TITLE:

\title{
Extraction of cellulose-synthesizing activity of Gluconacetobacter xylinus by alkylmaltoside.
}

\section{AUTHOR(S):}

Hashimoto, Akira; Shimono, Kenji; Horikawa, Yoshiki; Ichikawa, Tsukasa; Wada, Masahisa; Imai, Tomoya; Sugiyama, Junji

\section{CITATION:}

Hashimoto, Akira ...[et al]. Extraction of cellulose-synthesizing activity of

Gluconaceto bacter xylinus by alkylmaltoside.. Carbohydrate research 2011, 346(17): 27602768

\section{ISSUE DATE:}

2011-12-13

URL:

http://hdl.handle.net/2433/151721

\section{RIGHT:}

(C) 2011 Elsevier Ltd.; This is not the published version. Please cite only the published version.; この論文は出版社版でありません。引用の際に は出版社版をご確認ご利用ください。 


\title{
Extraction of cellulose-synthesizing activity of Gluconacetobacter xylinus by alkylmaltoside
}

\author{
Akira Hashimoto $^{\mathrm{a}, \uparrow, \S}$, Kenji Shimono $^{\mathrm{a}, \S}$, Yoshiki Horikawa ${ }^{\mathrm{a}}$, Tsukasa Ichikawa ${ }^{\mathrm{a}, \grave{\hbar}}$, Masahisa Wada ${ }^{\mathrm{b}, \mathrm{c}}$, \\ Tomoya Imai ${ }^{\mathrm{a}, *}$, Junji Sugiyama ${ }^{\mathrm{a}}$ \\ ${ }^{a}$ Research Institute of Sustainable Humanosphere (RISH), Kyoto University, Uji, Kyoto 611-0011, Japan \\ ${ }^{\mathrm{b}}$ Graduate School of Agricultural and Life Sciences, The University of Tokyo, Yayoi 1-1-1, Bunkyo-ku, Tokyo 113-0011, Japan \\ ${ }^{\mathrm{c} D e p a r t m e n t}$ of Plant \& Environmental New Resources, College of Life Sciences, Kyung Hee University, 1, Seocheon-dong, Giheung-ku, Yongin-si, Gyeonggi-do 446-701, \\ Republic of Korea
}

\section{A R T I C L E I N F O}

\section{Article history:}

Received 20 July 2011

Received in revised form 26 September

2011

Accepted 27 September 2011

Available online 4 October 2011

\section{Keywords:}

Cellulose synthase

CesA/BcsA

CesB/BcsB

Cellulose I

Gluconacetobacter xylinus

Membrane protein enzyme

\begin{abstract}
A B S T R A C T
This study reinvestigated the synthesis of cellulose in vitro with a well-known cellulose-producing bacterium, Gluconacetobacter xylinus. Alkylmaltoside detergents, which are more frequently used in recent structural biological researches, are uniquely used in this study to solubilize cellulose-synthesizing activity from the cell membrane of G. xylinus. Activity comparable to that previously reported is obtained, while the synthesized cellulose is crystallized into a non-native polymorph of cellulose (cellulose II) as well as the previous studies. In spite of this failure to recover the native activity to synthesize cellulose I microfibril in vitro, the product is a polymer with a degree of polymerization greater than 45 as determined by matrix-assisted laser desorption/ionization time-of-flight mass spectrometry (MALDI-TOFMS). It was thus concluded that the established protocol can solubilize cellulose-synthesizing activity of $G$. xylinus with polymerizing activity.
\end{abstract}

(c) 2011 Elsevier Ltd. All rights reserved.

\section{Introduction}

Living organisms produce up to 10 billion tons of cellulose each year, and cellulose is considered to be the most promising source of biorefineries and biofuel. Such massive cellulose production means that the ability to synthesize cellulose is beneficial to the organisms that acquired this ability through evolution. This may be because cellulose naturally exists as a nanofiber, the cellulose microfibril. The most striking example will be seen in plant cell walls, where the microfibrils can create a framework rigid enough to support a $100-\mathrm{m}$ tall tree.

Therefore, it is very clear that the cellulose synthesizing enzyme, cellulose synthase, must not only polymerize glucose residues but also spin the polymeric $\beta$-glucan chain into a microfibril. Such a complicated process is realized by the cellulose synthase complex, which is visualized as the terminal complex (TC) at the tip of the cellulose microfiber on cell membranes. ${ }^{1-3}$ Consistent with this observation, cellulose synthesizing activity is

\footnotetext{
* Corresponding author. Tel.: +81 77438 3631; fax: +81 774383635 .

E-mail address: timai@rish.kyoto-u.ac.jp (T. Imai).

Present address: Rengo Co., Ltd., Ryoke 5-14-8, Kawaguchi-shi, Saitama 332-0004, Japan.

$\$$ Present address: Ministry of Agriculture, Forestry and Fisheries, Kasumigaseki 1-2-1, Chiyoda-ku, Tokyo 100-8950, Japan.

$\S$ These authors equally contributed to the work.
}

biochemically located in the cell membrane, ${ }^{4,5}$ and the gene of the catalytic subunit, cesA, encodes transmembrane (TM) protein with eight putative TM helices. ${ }^{6,7}$ In addition, many studies have suggested that cellulose synthase is a hetero-subunit complex. ${ }^{7}$

A bacterial homologue often plays an important role for studying complicated membrane proteins, ${ }^{8-10}$ because it is easier to handle and functions well enough to help understand their key functions. For studying cellulose biosynthesis, Gluconacetobacter xylinus (formerly Acetobacter xylinum), a Gram-negative bacterium, has been a popular model organism. Indeed, the first ces $A$ gene was found in this bacterium, ${ }^{11,12}$ and originally named $b c s A$ (bacterial cellulose synthase A); hereafter in this article, we will refer to bcs in G. xylinus as gxces according to the proposed nomenclature convention. ${ }^{6}$ The gxces $A$ gene is followed by three open reading frames, gxces $B$, gxces $C$, and gxces $D$ (formerly $b c s B, b c s C$, and $b c s D$, respectively), which form an operon and are thought to function as a complex with gxcesA. ${ }^{12,13}$ Furthermore, cellulose-synthesizing activity can be isolated from $G$. xylinus, which actually allowed for the purification of cellulose synthase proteins, GxCesA and GxCesB. ${ }^{14,15}$ Mutagenesis studies of $G$. xylinus demonstrated that nonfunctional mutants of both GxCesC and GxCesD can synthesize cellulose in vitro but not in vivo. ${ }^{12}$ These data show that GxCesA and GxCesB proteins are necessary subunits for cellulose biosynthesis.

We reinvestigated in vitro cellulose biosynthesis using G. xylinus as an experimental model. Particularly alkylmaltoside was 
tested as a detergent to solubilize the cellulose-synthesizing activity in the cell membrane. An alkylmaltoside has been used more frequently in recent structural biological researches of membrane proteins, ${ }^{16}$ and should be of help in our structural biological studies of cellulose synthase. We first checked the cellulose-synthesizing activity in the alkylamaltoside extract from the cell membrane in comparison with the previously used detergents, digitonin and Triton X-100. Then the in vitro synthesized cellulose was analyzed by an electron microscope, by electron/X-ray diffraction, by Fourier-transform infrared (FTIR) spectroscopy and by matrix-assisted laser desorption/ionization time-of-flight mass spectrometry (MALDI-TOFMS).

\section{Results}

\subsection{Modification of the in vitro synthesis of cellulose with G. xylinus}

In previous studies, TME buffer ( $50 \mathrm{mM}$ Tris- $\mathrm{HCl}(\mathrm{pH} 7.5)$, $10 \mathrm{mM} \mathrm{MgCl} 2$, and $1 \mathrm{mM}$ EDTA) was used together with PEG-4000 to isolate the cellulose-synthesizing activity. ${ }^{4,17}$ PEG is a protein precipitant, and may bring unrelated proteins during purification that interfere with subsequent experiments. Thus, we removed PEG from the buffer to allow for further analysis of the obtained cellulose-synthesizing activity. In addition, the buffer in this study was prepared with 3-morpholinopropane sulfonic acid (MOPS), a buffer introduced by Good and colleagues in the 1960s, which recent studies used to improve results. For example, recombinant cotton CesA protein can synthesize longer cell-oligosaccharides in MOPS buffer than in Tris buffer. ${ }^{18}$ As some of Good's buffers like MOPS or PIPES (1,4-piperazinediethane sulfonic acid) were used for in vitro cellulose synthesis, ${ }^{19-22}$ we expected that Good's buffers will improve the isolation of cellulose-synthesizing activity from G. xylinus. Thus, we used MOPS-buffer without PEG to extract cellulose-synthesizing activity from $G$. xylinus membrane by detergent.

To solubilize the cellulose-synthesizing activity in this study, we checked six detergents including the alkylmaltosides, $n$-decyl$\beta$-maltoside (DM), $n$-undecyl $\beta$-maltoside (UDM), and $n$-dodecyl $\beta$-maltoside (DDM), as well as 1,2-diheptanoyl-sn-glycero-3-phosphocholine (DHPC), together with the previously used detergent digitonin and Triton $\mathrm{X}-100^{14,17}$ for comparison. The concentration of detergent was optimized roughly between $0.2 \%$ and $2.0 \%$. The reaction was conducted for $1 \mathrm{~h}$ to count the activity until the synthesizing-reaction almost stopped. As shown in Table 1, the specific activity of any detergent extract is significantly higher than that of membrane $(P<0.05$ at least). Among these detergents, the alkylmaltoside is very valuable because it is one of the promising detergents for membrane proteins in structural biology, ${ }^{16}$ and it is shown that PEG-free buffer is available to isolate the activity. Thus this protocol should be quite useful for studying cellulose

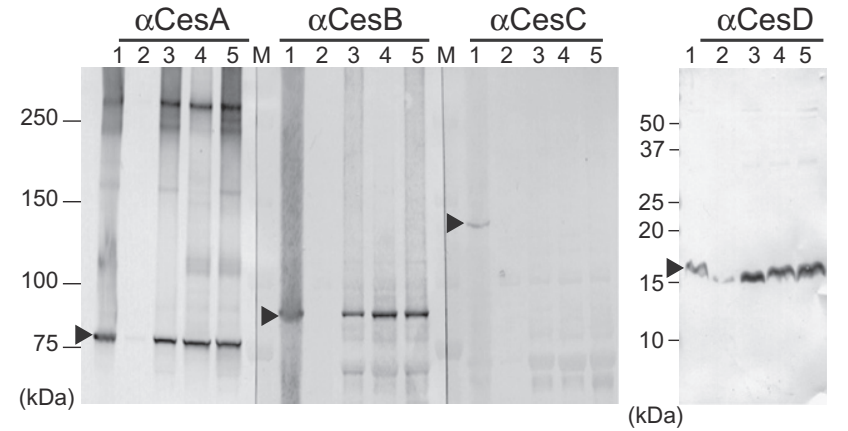

Figure 1. Western blotting of detergent extracts. Detergent extracts were subjected to SDS-PAGE on a precast 5-20\% gradient polyacrylamide gel (SuperSep, Wako Pure Chemicals Industries Ltd.) for detecting GxCesA, GxCesB and GxCesC, and 15\% labmade polyacrylamide gel for GxCesD. The first antibodies against GxCesA, GxCesB, GxCesC and GxCesD were visualized with alkaline phosphatase-conjugated secondary antibody. The $\boldsymbol{D}$ indicates the band of each protein. Lane 1: membrane fraction; lane 2: digitonin extract; lane 3: Triton X-100 extract; lane 4: n-decyl$\beta$-maltoside (DM) extract; lane 5: $n$-dodecyl- $\beta$-maltoside (DDM) extract; lane M: molecular weight marker

synthase in detail, like structural biology, enzymatic analysis, and other direct studies of cellulose synthase.

The rise in specific activity through the biochemical process is, in many cases, attributed to the enrichment of activity or related protein(s). Nevertheless, a careful survey of the data does not support that. The increase in specific activity is probably accounted for by the increase of 'total activity' by solubilization (except for digitonin and DHPC) as shown in Table 1. It is very surprising that solubilization gives rise to more total activity than does the membrane, because solubilization in general prepares less protein than does the membrane. The most likely interpretation is that solubilization enhances the cellulose-synthesizing activity in the cases of Triton X-100, DM, and DDM. In contrast, digitonin and DHPC provide higher specific activities without significant increase of total activity, implying that the enrichment of enzymatic activity by these detergents is more evident.

\subsection{Electrophoresis analysis}

As shown by the activity assay, digitonin and DHPC give rise to less cellulose-synthesizing activity in the extract in spite of its selective solubilization. Western blotting clearly supported this result, showing that the digitonin extract has less GxCesA and GxCesB proteins, the subunits necessary for in vitro cellulosesynthesizing activity of $G$. xylinus. On the other hand, more GxCesA and GxCesB are found in the detergent extract of Triton X-100, DM, and DDM, which has more total activity as shown in Table 1 . Thus it is supposed that GxCesA and GxCesB proteins are necessary for

Table 1

Total and specific activity of cellulose synthesis in vitro by G. xylinus

\begin{tabular}{|c|c|c|c|c|c|}
\hline \multirow[t]{2}{*}{ Sample } & \multicolumn{2}{|c|}{ Specific activity (nmol of glucose/min/mg of protein) } & \multicolumn{2}{|c|}{ Total activity (nmol of glucose/min/assay) } & \\
\hline & Mean \pm S.D. & Statistical significances from membrane & Mean \pm S.D. & Statistical significance from membrane & \\
\hline Membrane & $0.67 \pm 0.16$ & - & $0.28 \pm 0.12$ & - & $(n=15)$ \\
\hline 1.0\% digitonin & $2.7 \pm 0.52$ & $(P<0.01)$ & $0.29 \pm 0.16$ & N.S. $(P=0.92)$ & $(n=4)$ \\
\hline $1.0 \%$ Triton $\mathrm{X}-100$ & $3.3 \pm 1.1$ & $(P=0.017)$ & $0.75 \pm 0.2$ & $(P=0.014)$ & $(n=4)$ \\
\hline $2.0 \% \mathrm{DM}$ & $5.0 \pm 2.4$ & $(P<0.01)$ & $1.1 \pm 0.41$ & $(P<0.01)$ & $(n=10)$ \\
\hline $1.5 \% \mathrm{UDM}$ & $2.9 \pm 0.39$ & $(P<0.01)$ & $0.52 \pm 0.03$ & $(P<0.01)$ & $(n=4)$ \\
\hline 1.0\% DDM & $3.9 \pm 2.3$ & $(P<0.01)$ & $0.93 \pm 0.58$ & $(P<0.01)$ & $(n=10)$ \\
\hline $0.5 \%$ DHPC & $1.3 \pm 0.31$ & $(P<0.01)$ & $0.26 \pm 0.09$ & N.S. $(P=0.73)$ & $(n=6)$ \\
\hline
\end{tabular}

Total activity is compared with each other assay by assay of a $0.2 \mathrm{~mL}$ sample. All results are shown as mean \pm S.D. The results of statistical analyses are shown in the column of statistical significances: Welch's $t$-test was performed between membrane and each detergent extract, and their $P$-values are indicated for each. N.S. stands for 'not significant'. 
the in vitro activity of cellulose synthesis, and probably form a complex together in G. xylinus. Western blotting with antibodies against GxCesC and GxCesD showed that the former is not solubilized and the latter is solubilized, whichever detergent is used in this study, especially Triton X-100, DM, and DDM, as well as GxCesA and GxCesB.

\subsection{Structural analyses revealed that the in vitro product is cellulose II}

In this study, we conducted structural analyses mainly for the cellulose synthesized by DM and DDM extracts because of sufficient amounts of samples, although electron microscopy with
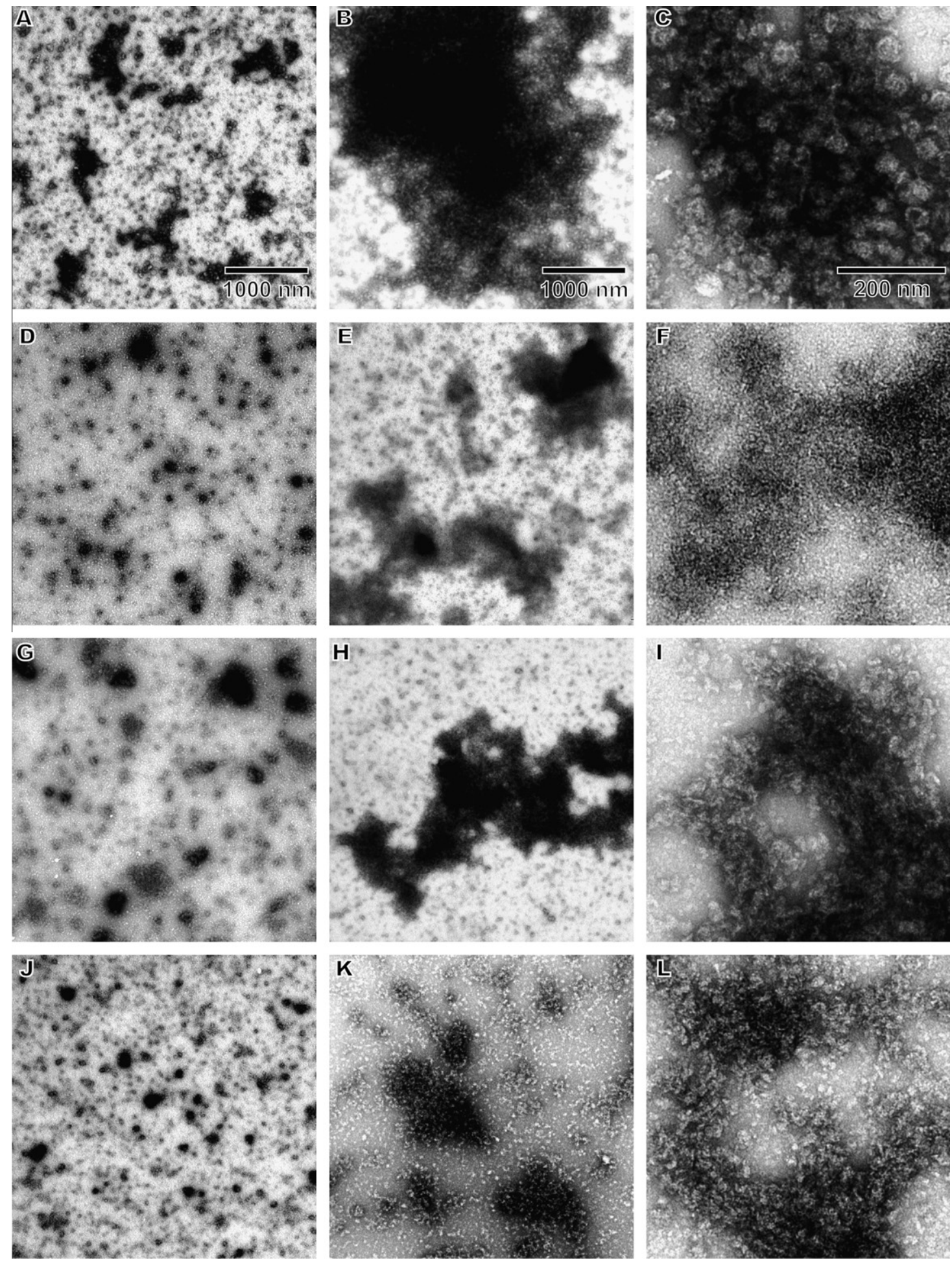

Figure 2. Electron micrographs of cellulose synthesized in vitro. Reaction was directly stained by uranyl acetate for negative staining. The detergent used for solubilization is, respectively, digitonin, Triton X-100, DM, and DDM in A-C, D-F, G-I, and J-L. Micrographs A, D, G, and J show the reactions without UDP-glucose substrate, while B, E, H and K do those with UDP-glucose. C, F, I, and L are close-up views of the reactions with UDP-glucose. 

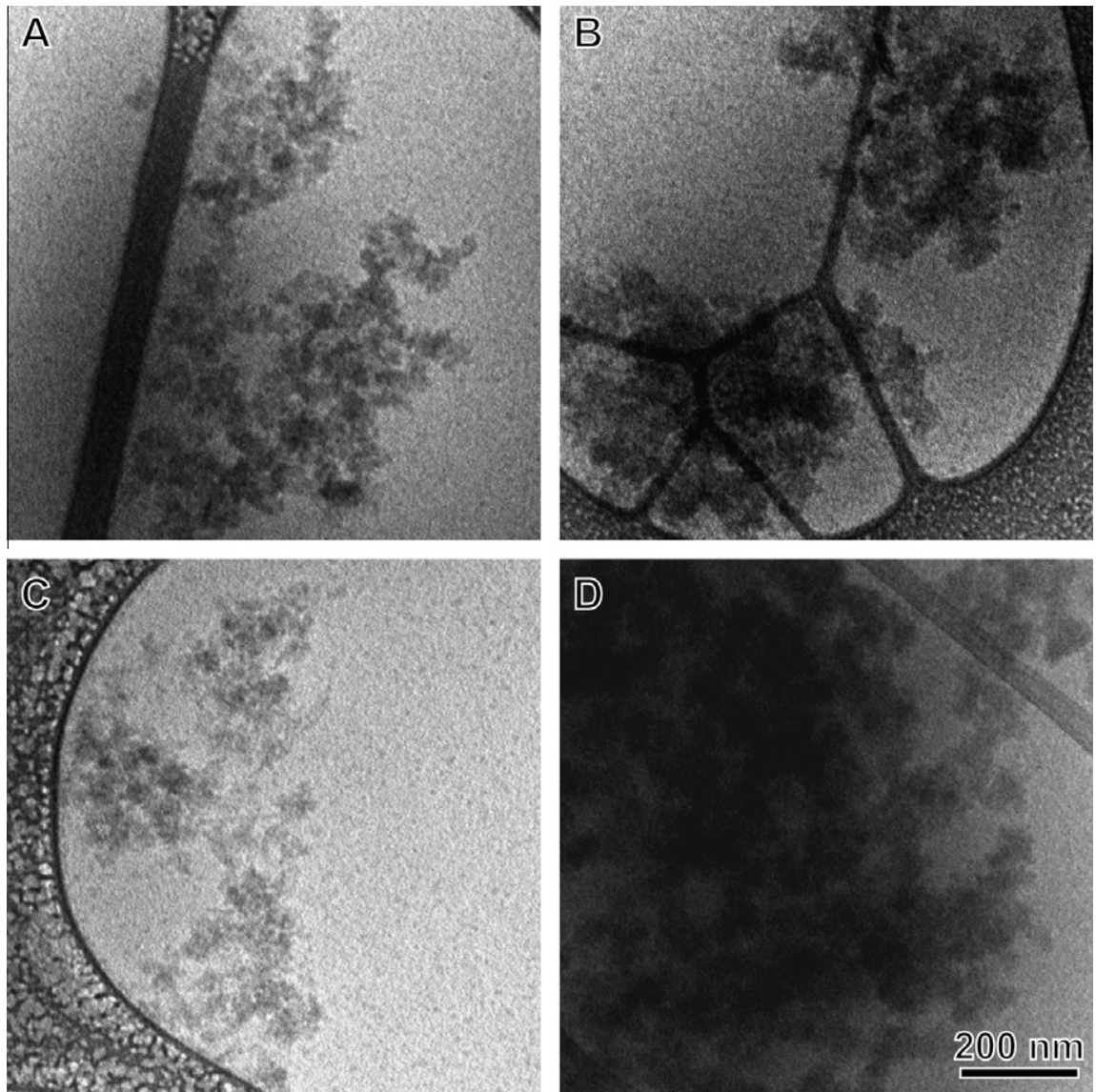

Figure 3. Flash-frozen reaction of in vitro cellulose synthesis by DM (A and B) and DDM (C and D) extracts, observed by cryo-electron micrographs. The reaction conducted without UDP-glucose is shown in A and C, showing less dense aggregation than the reaction with UDP-glucose shown in B and D.

negative staining was carried out for all the detergents. Electron micrographs with negative staining clearly showed that the products synthesized in vitro are not in the form of microfibrils, but are aggregates of particles approximately $30 \mathrm{~nm}$ in size (Fig. 2). These globular aggregates are also observed by cryo-electron microscopy in flash-frozen vitreous reaction samples (Fig. 3). The dense part at the center of the aggregation seems to be cellulose aggregates, while the peripheral part is less conclusive. The in vitro synthesized cellulose is unlikely to be aggregated by drying, but likely it exists as an aggregation already when released from enzyme in aqueous solution. Electron diffraction diagrams of these products are shown in Figure 4, and two diffraction rings corresponding to 0.45 and $0.41 \mathrm{~nm} d$-spacings are typically observed in both the DM and DDM samples. These values are somewhat larger than $d$ spacings of 110 and 020 diffraction calculated from the unit cell of cellulose $\mathrm{II}^{23}$ and the other major equatorial diffraction of $1 \overline{1} 0$ ring is not visible. Nonetheless, the pattern is explained as that of cellulose II, and wide-angle X-ray diffraction and FTIR spectroscopy support this interpretation (see below).

X-ray diffraction and FTIR spectroscopy provide an averaged view of the structure, and both clearly showed that the product synthesized in vitro is cellulose II when Triton X-100, DM, and DDM are used. Three major diffraction peaks observed by X-ray diffraction are clearly characteristic of cellulose II, each of which corresponds to $1 \overline{1}$ 0,110 and 020 (Fig. 5A and Table 2). As well, FTIR spectroscopy clearly shows substantially the same spectrum as mercerized bacterial cellulose, indicating that the product is cellulose II (Fig. 6). Thus, our study confirmed that a non-native form of cellulose II is produced even when an alkylmaltoside is used for solubilizing the activity from the cell membrane, as well as classi-
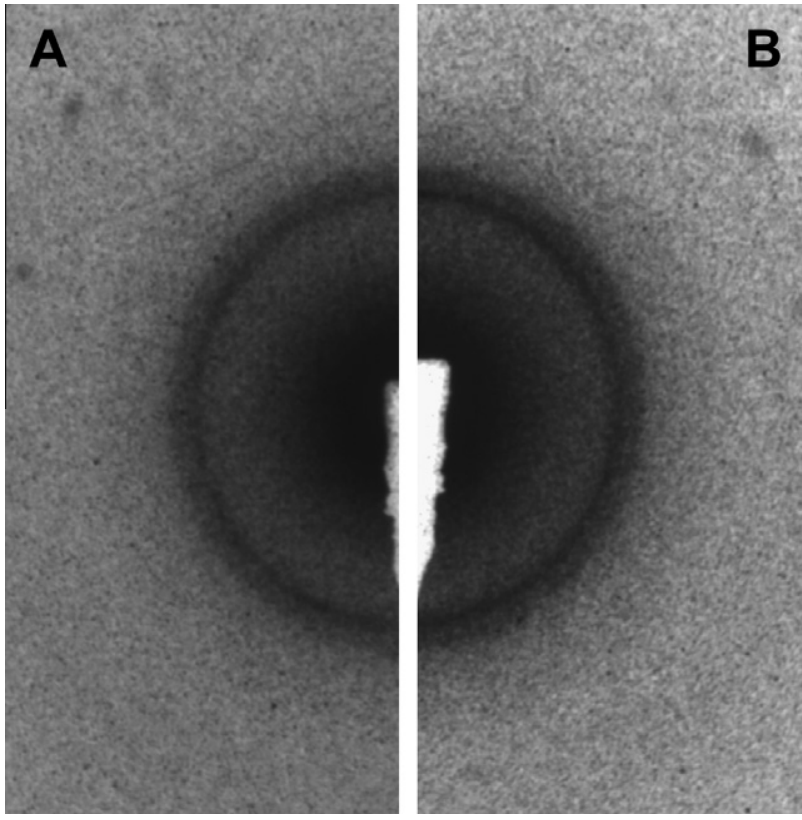

Figure 4. Electron diffraction diagrams of cellulose synthesized in vitro by DM (A) and DDM (B) extracts. Clear and diffused ring are observed, corresponding to $0.45 \mathrm{~nm}$ and $0.41 \mathrm{~nm} d$-spacings, respectively, and to (110) and (020) planes of cellulose II. The $1 \overline{1} 0$ diffraction that should appear as the innermost ring is invisible probably because of the crystallographic orientation.

cal detergents in the previous studies. ${ }^{17,24}$ Accordingly, it is now clearly shown that electron diffraction diagrams in Figure 4 result 
A

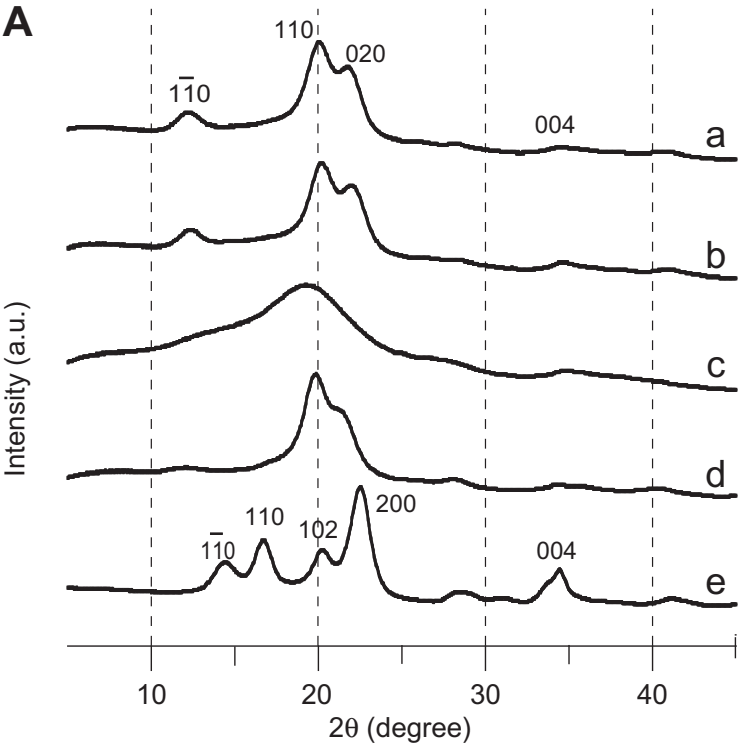

B

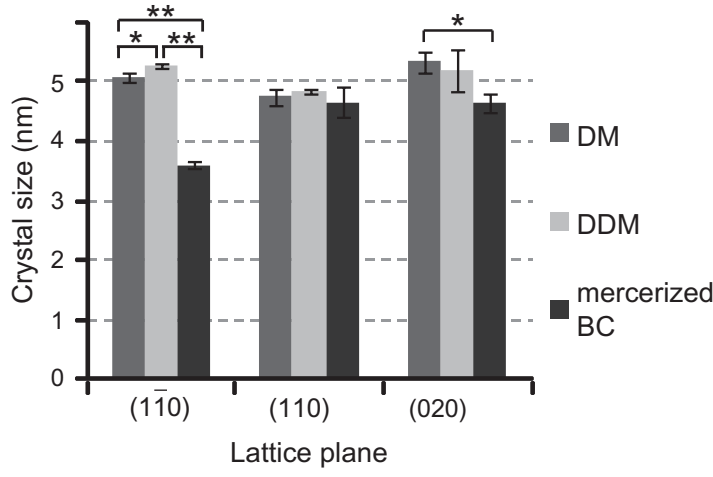

Figure 5. (A) X-ray diffraction curves of the lyophilized products. Cellulose synthesized in vitro by DM (a) and DDM (b) extracts. In (c) and (d), regenerated and mercerized cellulose prepared from BC is, respectively, shown. In (e), origina bacterial cellulose of G. xylinus is shown. Diffraction curves (a)-(d) are clearly interpreted and indexed as cellulose II, whereas the curve (e) indexed as cellulose I. (B) The width of the equatorial lattice is compared between the in vitro product and the mercerized cellulose. The pair significantly different from each other is marked by asterisk(s): single and double asterisks mean $P<0.05$ and $P<0.01$, respectively.

from a cellulose II crystal with a preferential orientation, which brings the $(1 \overline{1} 0)$ plane out of the Bragg condition while keeping (110) and (020) in the condition. This orientation rules out the possibility that cellulose chains are packed perpendicularly in the product, and suggests that the chains are laid in the product. Thus the in vitro synthesized cellulose by detergent extract is quite different from the enzymatically synthesized cellulose by reversed reaction or glycolsynthase,,$^{25-27}$ in which the perpendicularly packed cellulose chains are often found.

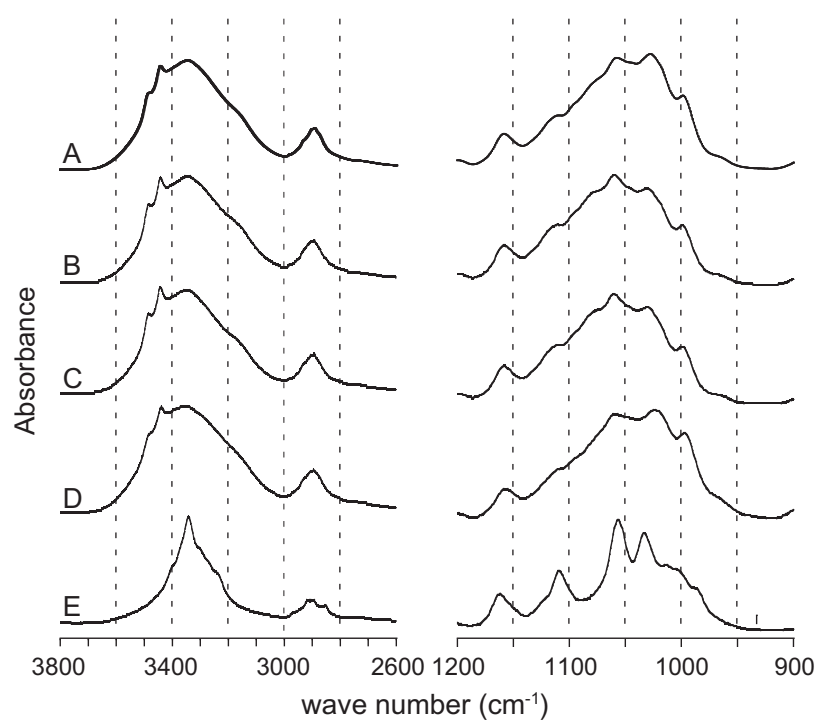

Figure 6. FTIR spectra of cellulose synthesized in vitro by Triton X-100-(A), DM(B) and DDM-(C) extract. D, mercerized bacterial cellulose. E, original bacterial cellulose synthesized in vivo by G. xylinus. Two characteristic spectral regions are shown: OH-stretching $\left(3600-3000 \mathrm{~cm}^{-1}\right)$ and the fingerprint region $(1200$ $\left.900 \mathrm{~cm}^{-1}\right)$.

In this study, two standard samples of cellulose II are prepared from the bacterial cellulose (BC) of G. xylinus: regenerated and mercerized cellulose. These two samples, both of which gave typical X-ray diffraction of cellulose II (Fig. 5A, c, and d), show different diffraction profiles from each other, reflecting differences of the fine structure and its crystallization mechanism. X-ray diffraction of the in vitro synthesized cellulose from either the DM or DDM extract is much sharper than that from the regenerated cellulose, and it is fairly sharper than the mercerized example (Fig. 5A). Careful comparison with the mercerized $B C$ showed that the crystal size of the $(1 \overline{1} 0)$ plane of the in vitro product was clearly larger than that of the mercerized sample (Fig. 5B and Table 2). FTIR spectroscopy also showed that the cellulose synthesized in vitro gives slightly but significantly sharper spectra than those of the mercerized $\mathrm{BC}$, especially in the $\mathrm{OH}$-stretching region $\left(3000-3600 \mathrm{~cm}^{-1}\right)$, indicating that hydrogen bonding is better ordered in the in vitro synthesized cellulose. This is quite consistent with the stronger $1 \overline{1} 0$ ring observed by X-ray diffraction, since highly ordered hydrogen bonding that stacks the molecular sheets corresponding to the $(1 \overline{1} 0)$ plane would produce such results.

\subsection{MALDI-TOFMS revealed that the in vitro product is a polymer rather than an oligomer}

MALDI-TOFMS is a commonly used method for analyzing polymers, including polysaccharides. ${ }^{28,29}$ The mass spectrum of the

Table 2

$d$-Spacings and crystal size of equatorial lattices of cellulose II synthesized in vitro and mercerized bacterial cellulose, estimated by X-ray diffraction

\begin{tabular}{|c|c|c|c|c|}
\hline & & Product by DM-extract & Product by DDM-extract & Mercerized BC \\
\hline \multicolumn{5}{|c|}{$h k l$-index } \\
\hline \multirow{2}{*}{$(1 \overline{1} 0)$} & $d$-spacings $(\mathrm{nm})$ & $0.721(0.027)$ & $0.721(0.027)$ & $0.726(0.062)$ \\
\hline & Crystal size (nm) & $5.1(0.06)$ & $5.3(0.05)$ & $3.6(0.05)$ \\
\hline \multirow[t]{2}{*}{$(110)$} & $d$-spacings $(\mathrm{nm})$ & $0.441(0.013)$ & $0.441(0.018)$ & $0.444(0.034)$ \\
\hline & Crystal size (nm) & $4.7(0.15)$ & $4.8(0.03)$ & $4.6(0.25)$ \\
\hline \multirow[t]{2}{*}{$(020)$} & $d$-spacings $(\mathrm{nm})$ & $0.405(0.011)$ & $0.404(0.021)$ & $0.410(0.031)$ \\
\hline & Crystal size (nm) & $5.3(0.18)$ & $5.2(0.34)$ & $4.6(0.17)$ \\
\hline
\end{tabular}

The standard deviation is indicated in parentheses. 


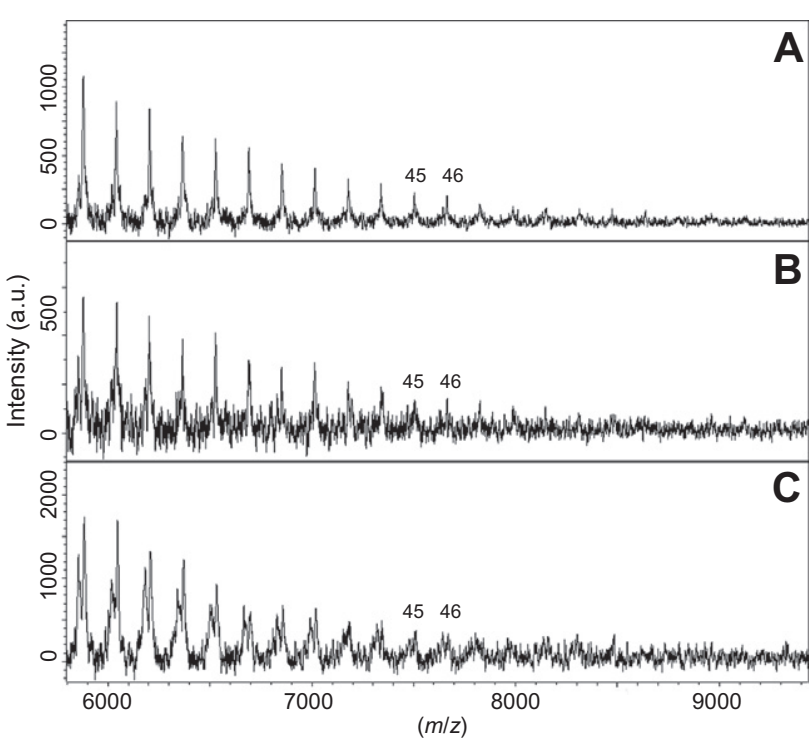

Figure 7. MALDI-TOFMS taken from cellulose synthesized in vitro with DM-(A) and DDM-(B) extracts, and cellulose II prepared by alkaline treatment of commercially available cellulose Avicel PH-101 (C). The number of DP is indicated above peaks of the spectra. Two peaks are observed in a single cluster of the spectrum, which is most obvious in C. These peaks are interpreted as the sodium adduct (higher mass) and the dehydrated sodium adduct (lower mass), respectively.

in vitro synthesized cellulose by MALDI-TOFMS shows a dehydrated glucose series (interval of $m / z 162$ ) as a sodium adduct, and sometimes also as the dehydrated sodium adduct, clearly demonstrating that the product is a glucan (Fig. 7). Although a whole distribution of DP could not be seen, a series of signals was seen up to $m / z 7500$, corresponding to DP 45 of a glucan. The mass spectrum from mercerized Avicel PH-101 was also significant up to $m / z$ 7500. The DP of Avicel PH-101 is approximately $200,{ }^{30}$ strongly indicating that the MALDI conditions used in this study cannot measure masses higher than the DP 45 of cellulose. However, the DP 45 sample is already much higher than those synthesized by the reversed reaction of degrading enzymes. ${ }^{26,27,31,32}$ It will be very important to verify whether the DP of the in vitro synthesized cellulose is as high as 3000 , the value of native bacterial cellulose, ${ }^{33}$ for example, by viscosity measurements.

\section{Discussion}

This study clearly showed two meaningful modifications of the protocol for extracting the cellulose-synthesizing activity in G. xylinus: (i) removal of PEG from the buffer and (ii) solubilization of the membrane proteins by an alkylmaltoside. PEG precipitates macromolecules including anything unrelated in the system, and it is definitely not preferable for further analyses. Alkylmaltoside is the most frequently used detergent for protein purification to crystallize membrane proteins, ${ }^{16}$ so less interference with cellulosesynthesizing activity is expected. Thus, the system reported in this study is very preferable for studying cellulose biosynthesis in detail, for example from the standpoint of immuno-precipitation and structural biology.

Cellulose synthase is known to form a complex in the cell membrane, and it is visually observed by freeze replica electron microscopy as a linear type TC in G. xylinus. ${ }^{34}$ Such an ordered arrangement of proteins usually requires specific inter- and/or intra-subunit interactions, and could be disintegrated in mixed micelles after detergent solubilization. Thus the alteration of cellulose synthase activity would be two-fold: disintegration of the complex and release from the lipid bilayer. Consequently cellulose synthesis in the presence of detergent is less controlled, leading to the formation of cellulose II, the most thermodynamically stable crystallographic polymorph of cellulose, ${ }^{35}$ instead of spinning a cellulose I microfibril. The apparent enhancement of activity by detergent solubilization, as observed in this study, may result from less control of the enzyme or 'partial denaturation'. In other words, free energy will be necessary for the spinning function. We believe that the spinning function is actually a part of cellulose synthase, and it must be realized with a supermolecular complex of membrane proteins.

Important subunits for either the spinning or crystallization step will be GxCesC and GxCesD as discussed previously. ${ }^{13} \mathrm{X}$-ray crystallographic analysis clearly shows that GxCesD is a dimer of two tetrameric subunits through which four cellulose chains are likely to pass, ${ }^{36}$ and a nonfunctional gxcesD mutant of G. xylinus synthesizes less cellulose with irregular fiber formation and crystallization in vivo. ${ }^{12,13,36}$ This protein was heterogeneously expressed as a soluble protein in this X-ray crystallographic study, and does not contain an apparent hydrophobic patch in its amino acid sequence. Nevertheless as shown by Western blotting in Figure 1, GxCesD is isolated together with the membrane, and the partial denaturation (cellulose II formation) occurs in the presence of GxCesD in the detergent extract (Fig. 1). These data mean that GxCesD may be incorporated in cellulose synthase complex by protein-protein interaction with any of the GxCesA, GxCesB, GxCesC or other subunits, which will be easily disintegrated. Considering that $\mathrm{GxCesC}$ is not solubilized in the detergent extract in this study (Fig. 1), GxCesC might link GxCesD with the GxCesAB complex. GxCesC is hypothesized to extrude synthesized cellulose chains, ${ }^{13}$ and probably functions concertedly with GxCesD. Thus GxCesC is one of the candidates for the missing piece(s) to reconstruct the full complex of $G$. xylinus cellulose synthase in vitro, as well as the reconstitution in the lipid membrane.

Crystallization is considered to be an important step in cellulose biosynthesis. Then it is very interesting that the cellulose II synthesized in vitro in this study has higher crystallinity than regenerated and mercerized BC. Regenerated cellulose is crystallized from the solution state, and is thus considered as a typical example of the crystallization in thermodynamic equilibration, and then the more ordered molecular arrangement in the in vitro cellulose II, shown by X-ray diffraction (Fig. 5A), is very characteristic. When compared with the mercerized cellulose, the crystal size of $\left(\begin{array}{ll}1 & 1\end{array}\right)$ plane is larger than that of cellulose II prepared by mercerizing the original $\mathrm{BC}$, whereas the difference is less clear in the other equatorial lattice planes (110) and (020) (Fig. 5B). The (1 $\overline{1} 0)$ plane is virtually a molecular sheet stacked by hydrophobic interactions, and then pulled together by hydrogen bonding to form cellulose II crystallite. ${ }^{23,37}$ Then more ordered hydrogen bonding observed in the IR spectra will be associated with larger number of $(1 \overline{1} 0)$ lattice planes in the crystal, which accounts for the observed larger crystal size of $(1 \overline{1} 0)$. Whatever the mechanism is, the crystallization that occurs in the in vitro synthesis in this study is quite unique and completely different from those seen in regeneration or mercerization of cellulose.

Comparisons with artificial cellulose synthesis show how unique the polymerizing activity is. Several studies have shown that the artificial synthesis of cellulose (not cellulose derivatives) is possible by reversing the degradation reaction performed by hydrolases and phosphorylases. ${ }^{25-27,31,32}$ Notably, the cellulose synthesized by this method has a DP of 10-20, clearly shorter than the cellulose synthesized in this study. This observation is very insightful, since there are no substantial differences in the chemical mechanisms between these two systems: glucose/cellobiose is successively linked when a leaving group at $\mathrm{C} 1$ of the sugar is in proximity to the acceptor cellulose molecule. This means that the 
native cellulose-synthesizing activity is very specialized for growing the cellulose chain longer, probably by holding the acceptor cellulose molecule in place during the reaction. This is known as processivity, for which the D,QXXRW motif is hypothetically responsible in CesA and other polysaccharides synthases. ${ }^{38,39}$ The exact function of this motif remains to be clarified.

It should be noted that the formation of the cellulose I fibrillar structure has been observed in vitro when plants were used in spite of solubilization by detergent. ${ }^{5,19,40}$ Thus, it appears that the plant cellulose synthase complex (or TC) remains intact even after solubilization, suggesting that plant is a more promising model for studying cellulose biosynthesis. G. xylinus is still a good model for directly studying cellulose biosynthesis because of its abundant and uncontaminated cellulose synthesizing activity. In plants, large amounts of callose ( $\beta$-( $1 \rightarrow 3)$-D-glucan) are synthesized in vitro from UDP-glucose and obscure the cellulose-synthesizing activity. ${ }^{5,19,40}$ This makes it difficult to use plants as models for studying cellulose biosynthesis.

The amino acid sequence of the GxCesA protein bears little homology to plant CesA even in the conserved regions: $25 \%$ identity and $40 \%$ similarity to the conserved regions of AtCesA1 (Arabidopsis thaliana), and $10 \%$ identity in the overall sequence. In addition, homologues of GxCesB, GxCesC, and GxCesD are not found in plants or any other cellulose-producing organisms. It is, therefore, necessary to remember that the cellulose synthesis machinery can differ among species. But still it is attractive to use G. xylinus as a model because of its abundant and non-contaminated activity, especially if the system is improved so that the cellulose I microfibril is synthesized in vitro with recombinant proteins. One of the challenges will be the spinning mechanism of cellulose molecules into a microfibril. For silk protein, many studies have been reported to clarify its spinning mechanism, and it is shown that the molecules are in the liquid crystalline state in the gland before the spinning event. ${ }^{41}$ Cellulose biosynthesis would be quite important as another example of the spinning with less environmental impact, and G. xylinus will be a suitable model for understanding the detailed mechanism of simultaneous polymerization and crystallization in cellulose biosynthesis.

\section{Experimental}

\subsection{Materials}

The vinegar-producing, Gram-negative bacterium Gluconacetobacter xylinus was used to prepare membrane proteins for conducting cellulose synthesis in vitro. The strain used was ATCC53524, supplied by ATCC (American Type Culture Collection). Detergents used were purchased from Dojin, Inc. (Japan) for $n$-decyl- $\beta$-maltoside (DM) and $n$-dodecyl- $\beta$-maltoside (DDM), Anatrace, Inc. (USA) for $n$-undecyl- $\beta$-maltoside (UDM) and Triton X-100, Wako Chemicals Inc. (Japan) for digitonin, and Avanti Polar Lipids Inc. (USA) for 1,2diheptanoyl-sn-glycero-3-phosphocholine (DHPC).

Antibodies were prepared by immunizing rabbits with hemocyanin-conjugated synthetic peptide corresponding to the sequence of a part of each subunit. The sequence for the immunization was designed based on G. xylinus BPR2001: SGQTQEGKISRAAS for GxCesA (UniProt ID: O82859, the carboxyl-end), SPDLYTWRDRPNK for GxCesB (GenBank: BAA31464, the middle from 411 to $423 \mathrm{ami}-$ no acid), SGRYQKAGNWTESGA for GxCesC (UniProt ID: 082861, the carboxyl-end) and TRDIDAEDLNS for GxCesD (UniProt ID: P19451, the part corresponding to $\alpha 5$ in the model PDB $3 A J 1^{36}$ ). These sequences are almost the same as those of G. xylinus ATCC53264, the parent strain of ATCC53524 used in this study. The resultant antiserum was affinity purified with the peptide injected to the rabbit being immunized. Preparation of these anti- bodies was done by Peptide Institute, Inc. (Osaka, Japan) and Scrum, Inc. (Tokyo, Japan).

Avicel and bacterial cellulose (BC) were used for references. Avicel PH-101 was purchased from Fluka. BC was prepared by culturing G. xylinus in Schramm-Hestrin (SH) medium ${ }^{42}$ at $28{ }^{\circ} \mathrm{C}$ without shaking. A portion of these samples was mercerized by soaking them in $5 \mathrm{M} \mathrm{NaOH}$ at room temperature for $12 \mathrm{~h}$ and then washing. A small amount of $\mathrm{BC}$ was dissolved in $5 \mathrm{wt} \% \mathrm{LiCl}$ in $\mathrm{N}, \mathrm{N}$-dimethylacetamide solution with the concentration of about $0.1 \mathrm{wt} \%$ at $4{ }^{\circ} \mathrm{C}$. The cellulose solution was gently poured into an excess amount of methanol at room temperature, and thus the regenerated cellulose was successively washed with deionzied water. After freeze drying, these samples were used for analyses.

\subsection{Enzymatic synthesis of c-di-GMP}

c-di-GMP (cyclic-di-guanylmonophosphate), an allosteric effecter of the cellulose-synthesizing activity in G. xylinus, ${ }^{43,44}$ was enzymatically synthesized by using diguanyl cyclase (DGC). The DGC used in this study was from Shewanella oneidensis MR-1 (GeneBank ID: NP_717795; hereafter called SoDGC), found by Blastp-search as a homologue of the Vibrio cholerae protein previously shown to have in vitro DGC activity. ${ }^{45}$ The genomic DNA of S. oneidensis MR-1 was purchased from ATCC, and the sodgc gene was amplified by PCR using AmpliTaqGold (Applied Biosystems, Inc.). The PCR product was subcloned into pGEM-T easy vector (Promega Inc.), and its nucleotide sequence was verified before directionally inserting it into the $\mathrm{pBAD} / \mathrm{HisA}$ vector (Invitrogen Inc.) by restriction enzyme digestion and ligation.

Purification of recombinant SoDGC, enzymatic c-di-GMP synthesis, and c-di-GMP purification from the reaction mixture were done according to previously published protocols. ${ }^{43,45}$ Briefly, hexahistidine-tagged SoDGC was expressed by Escherichia coli TOP10 strain harboring the expression vector prepared as described above. The cells were lysed with a French cell press, and the lysate was clarified by centrifugation and subjected to $\mathrm{Ni}$ immobilized agarose (His-Select, Sigma-Aldrich, Inc.) to purify the recombinant DGC protein. GTP $(1 \mathrm{mM})$ was incubated with $0.1 \mathrm{mg} / \mathrm{mL}$ of SoDGC protein to convert GTP to c-di-GMP. The synthesized c-di-GMP was separated from the reaction mixture by anion-exchange chromatography with DEAE-Sepharose (GE Healthcare) and ammonium bicarbonate as the mobile phase. Each fraction was dried at $100^{\circ} \mathrm{C}$, and then dissolved in water. The fraction containing c-di-GMP was checked by MALDI-TOFMS (Autoflex III; Bruker Daltonics Inc.) and LC/MS/MS (LCMS-IT-TOF; Shimadzu Co. Ltd.), and its concentration was quantified by UV absorbance with an extinction coefficient of 24,700 at $252 \mathrm{~nm}$. ${ }^{44}$

\subsection{Biochemical isolation of cellulose synthesizing activity}

G. xylinus was cultured in $1.0 \mathrm{~L}$ of $\mathrm{SH}$ medium with $0.1 \%$ crude commercial cellulase (Celluclast $1.5 \mathrm{~L}$; Novozymes Inc.) at $28^{\circ} \mathrm{C}$ for $16-24 \mathrm{~h}$ until the culture reached $\mathrm{OD}_{660}$ of approximately 0.6 . Cultured cells were collected by centrifugation at $5000 \mathrm{~g}$ for $10 \mathrm{~min}$ at $4{ }^{\circ} \mathrm{C}$. The cells were suspended in cell suspension buffer (100 mM MOPS, pH 6.7; 2 mM EDTA; 2 mM EGTA) and crushed with a French cell press at 20,000 psi. The lysate was centrifuged at $5000 \mathrm{~g}$ for $10 \mathrm{~min}$ to pellet intact cells and debris, and the supernatant was ultracentrifuged at $100,000 \mathrm{~g}$ for $1 \mathrm{~h}$ at $4{ }^{\circ} \mathrm{C}$. The membrane pellet was resuspended in membrane suspension buffer (100 mM MOPS, pH 6.7; 2 mM EDTA; 2 mM EGTA; 10\% glycerol). Detergents were added to this suspension to solubilize the membrane protein and cellulose-synthesizing activity. The detergents used were $1 \%$ digitonin, $1 \%$ Triton X-100, $2 \%$ DM, $1.5 \%$ UDM, $1 \%$ $\mathrm{DDM}$, and $0.5 \%$ DHPC. After incubating at $4{ }^{\circ} \mathrm{C}$ for $30 \mathrm{~min}$, the detergent-containing membrane fraction was ultracentrifuged at 
$100,000 \mathrm{~g}$ for $1 \mathrm{~h}$; the supernatant was the detergent extract that contained solubilized cellulose-synthesizing activity. Protein concentrations in the detergent extracts were estimated by using the Protein Dc Assay Kit (Biorad, Inc.) with the sample diluted ten-fold, for ruling out any variable effects that depended on specimens.

\subsection{Electrophoresis}

SDS-PAGE was carried out with precast gel of 5-20\% gradient of acrylamide (Supersep 5-20\%, Wako Pure Chemicals, Inc., Japan) and freshly prepared gel of $15 \%$ acrylamide. Nitrocellulose membrane (Whatman PROTRAN, GE Healthcare Inc.) was used for transferring the electrophoresis pattern from the gel. The membrane after incubation with the first antibody was treated by the antibody against rabbit IgG conjugated with alkaline phosphatase (Promega, Inc.). Finally the band of interest was visualized by coloring substrates for alkaline phosphatase, NBT (nitro blue tetrazolium chloride), and BCIP (5-bromo-4-chloro-3-indolyl phosphate) (Promega, Inc.).

\subsection{In vitro cellulose synthesis}

In vitro cellulose synthesis with the detergent extract was performed with $1 \mathrm{mM}$ UDP-glucose, $10 \mu \mathrm{M}$ c-di-GMP, $8 \mathrm{mM} \mathrm{MgCl}$, $1 \mathrm{mM} \mathrm{CaCl}_{2}, 20 \mathrm{mM}$ cellobiose, $75 \mathrm{mM}$ MOPS (pH 6.7), 2.5\% glycerol, $0.5 \mathrm{mM}$ EDTA, and $0.5 \mathrm{mM}$ EGTA. For estimating the cellulose-synthesizing activity, UDP-D-[U- $\left.{ }^{14} \mathrm{C}\right]$ glucose was added to realize $200 \mu \mathrm{L}$ of the above reaction mixture with a specific radioactivity of $2.3 \mathrm{MBq} / \mathrm{mmol}$. The reaction was incubated for $1 \mathrm{~h}$ at room temperature without shaking and stopped by adding $1 \mathrm{~mL}$ of ethanol. The ethanol-insoluble product was filtered with a glass fiber filter (Whatman Grade GF/C glass microfiber filter; GE Healthcare), and the trapped radioactivity was quantified with a liquid scintillation counter (TriCarb-2800TR; Perkin-Elmer) in scintillation cocktail (Ultima Gold, Perkin-Elmer). The measured dissociation per minute (dpm) values were converted to the mole of glucose incorporated into the ethanol-insoluble product, or cellulose. The counting was carried out in duplicate. Statistical analysis was performed using Excel 2007 (Microsoft Inc.) with the add-in software Statcel2.

To obtain cellulose for analysis, the reaction was incubated for $18-24 \mathrm{~h}$ at room temperature without shaking. The white precipitate was directly transferred to electron microscopy support film for negative staining and cryo-electron microscopy. For the other analyses, the precipitate was collected by centrifugation and washed sequentially in $2 \%$ SDS and $2 \% \mathrm{NaOH}$ at $100^{\circ} \mathrm{C}$ for $1 \mathrm{~h}$. For electron diffraction, $2 \%$ SDS and $0.2 \% \mathrm{NaOH}$ were used at $70{ }^{\circ} \mathrm{C}$ for $1 \mathrm{~h}$ because the aggregate was too thick with the former condition. The precipitate was then washed thoroughly with water and centrifugation.

\subsection{Electron microscopy}

The reaction mixture was deposited onto carbon film supported by a copper grid that was glow-discharged just before use. For negative staining, $2 \%$ uranyl acetate solution was applied to the film and allowed to dry. For cryo-electron microscopy (cryo-EM), $3 \mu \mathrm{L}$ of the cellulose synthesis reaction mixture was taken out after 3-6 h and directly deposited onto freshly glow-discharged lacy carbon film on a copper grid (Ted Pella, Inc.). After removing the excess liquid by blotting with filter paper, the grid was quickly plunged into liquid ethane at $-175^{\circ} \mathrm{C}$ using KF-80 (Leica $\mathrm{GmbH}$ ) to obtain a vitreous sample. This flash-frozen reaction was transferred to a cryo-holder (626-DH; Gatan, Inc.) for observation. Electron micrographs of these samples were taken with a JEM-2000EX II (Jeol Co. Ltd.) microscope operated at $100 \mathrm{kV}$, and a side- mounted CCD camera (MegaView G2; Olympus Soft Imaging Solutions $\mathrm{GmbH}$ ).

The electron diffraction sample was deposited onto freshly glowdischarged carbon-coated copper grid. A JEM-2000EX II microscope was operated at an accelerating voltage of $100 \mathrm{kV}$. Electron microdiffraction was carried out with a $20-\mu \mathrm{m}$ condenser lens aperture inserted, and the first condenser lens was excited maximally (spot size 8). Diffraction diagrams were recorded at the shortest possible camera distance (nominally $15 \mathrm{~cm}$ ) on MEM film (Mitsubishi Paper Milling Co. Ltd.) and developed with correctol (Fujifilm Corp.) at $20^{\circ} \mathrm{C}$ for $6 \mathrm{~min}$. The diffractograms on film were digitized with a MegaPlus camera (Eastman Kodak, Inc.), and analyzed with ImageJ to calculate $d$-spacings from Debye rings; $0.2355 \mathrm{~nm}$ for (111) plane of Au was used as a reference for calibration.

\subsection{FTIR spectroscopy}

Spectrum One system (Perkin-Elmer. Inc.) was used in the attenuated total reflection (ATR) mode. The cellulose suspension was dried on a single-bound diamond probe of the ATR attachment, and the spectra were collected with a range of 4000$400 \mathrm{~cm}^{-1}$ by 16 times of integration. The spectra thus obtained were adjusted with baseline correction, smoothing, and intensity normalization.

\subsection{X-ray diffraction}

The washed cellulose samples were lyophilized and pressed to make tablets. X-ray diffraction diagrams were obtained using a vacuum camera mounted on a rotating anode X-ray generator (Rigaku RU-200BH). Ni-filtered $\mathrm{Cu} \mathrm{K} \alpha$ radiation $(\lambda=0.15418 \mathrm{~nm}$ ) generated at $50 \mathrm{kV}$ and $100 \mathrm{~mA}$ was collimated with a 0.3 mm-diameter pinhole. The collimated X-rays were directed at the samples, and ring X-ray patterns were recorded on imaging plates (BAS-IP SR 127, Fujifilm Corp.). The sample-to-imaging plate distance was calibrated using NaF powder $(d=0.23166 \mathrm{~nm})$. The size of the crystallite was estimated from the width at halfmaximum diffraction based on the Scherrer equation with $K=0.9$.

\subsection{MALDI-TOFMS}

An Autoflex III instrument (Bruker Daltonics Inc.) was operated in the linear-positive mode. $25 \mathrm{mg} / \mathrm{mL}$ of 2,5-dihydroxybenzoic acid (Bruker Daltonics Inc.) dissolved in $\mathrm{N}, \mathrm{N}$-dimethylformamide was used as the matrix; $1 \mu \mathrm{L}$ of the mixture was mixed with $1 \mu \mathrm{L}$ of the suspension of synthesized cellulose and air-dried on the stainless-steel target plate at room temperature. The operating protocol followed was the manufacturer's protocol for measuring the range of $2000-10,000 \mathrm{kDa}$. Laser power was adjusted in each sample to obtain ion counting on the order of $10^{4}$. Calibration was done with polyethylene glycol (PEG)-4000.

\section{Conclusions}

This study showed that cellulose-synthesizing activity in the $G$. xylinus cell membrane can be solubilized by an alkylmaltoside. This is very valuable information because this type of detergent should enhance the possibility of crystallizing the solubilized protein for crystallographic analysis. Although the synthesized cellulose was cellulose II rather than the native cellulose I microfibril as well as the previous studies, it is shown that the synthesized cellulose is a polymer by MALDI-TOFMS. These data suggest that the loss of the spinning function of $G$. xylinus cellulose synthase may be due to detergent solubilization of the lipid bilayer, and that the synthase itself is not severely damaged. 


\section{Acknowledgments}

LC/MS/MS analysis was carried out by the System for Development and Assessment of Sustainable Humanosphere (DASH), Research Institute for Sustainable Humanosphere (RISH), and Center for Ecological Research, Kyoto University. We thank Professor T. Watanabe and Dr. K. Yoshioka (RISH, Kyoto University) for MALDI-TOFMS analysis; Dr. H. Nishimura (RISH) for LC/MS/MS experiments; Professor K. Yazaki (RISH) for ultracentrifugation; and Professor Y. Fujiyoshi (Graduate School of Science, Kyoto University) for financial support to prepare polyclonal antibodies. This study is financially supported by a Grant-in-aid for Scientific Research A (19208017) and Grant-in-Aid for challenging Exploratory Research (22658053) to J.S., and a Grant-in-aid for Young Scientists A (23688021) to T.I.

\section{References}

1. Brown, M. R., Jr.; Montezinos, D. Proc. Natl. Acad. Sci. U.S.A. 1976, 73, 143-147.

2. Brown, M. R., Jr.; Willison, J. H. M.; Richardson, C. L. Proc. Natl. Acad. Sci. U.S.A 1976, $73,4565-4569$.

3. Giddings, T. H., Jr.; Brower, D. L.; Staehelin, L. A. J. Cell Biol. 1980, 84, 327-339.

4. Aloni, Y.; Cohen, R.; Benziman, M.; Delmer, D. J. Biol. Chem. 1983, 258, 4419-4423.

5. Okuda, K.; Li, L.; Kudlicka, K.; Kuga, S.; Brown, M. R., Jr. Plant Physiol. 1993, 101 1131-1142.

6. Delmer, D. P. Annu. Rev. Plant Biol. 1999, 50, 245-276.

7. Somerville, C. Annu. Rev. Cell Dev. Biol. 2006, 22, 53-78.

8. Doyle, D. A.; Cabral, J. M.; Pfuetzner, R. A.; Kuo, A.; Gulbis, J. M.; Cohen, S. L.; Chait, B. T.; MacKinnon, R. Science 1998, 280, 69-77.

9. Bass, R. B.; Strop, P.; Barclay, M.; Rees, D. C. Science 2002, 298, 1582-1587.

10. Cuello, L. G.; Cortes, D. M.; Perozo, E. Science 2004, 306, 491-495.

11. Saxena, I. M.; Lin, F. C.; Brown, M. R., Jr. Plant Mol. Biol. 1990, 15, 673-683.

12. Wong, H. C.; Fear, A. L.; Calhoon, R. D.; Eichinger, G. H.; Mayer, R.; Amikam, D.; Benziman, M.; Gelfand, D. H.; Meade, J. H.; Emerick, A. W.; Bruner, R.; BenBassat, A.; Tal, R. Proc. Natl. Acad. Sci. U.S.A. 1990, 87, 8130-8134.

13. Saxena, I. M.; Kudlicka, K.; Okuda, K.; Brown, M. R., Jr. J. Bacteriol. 1994, 176 5735-5752.

14. Lin, F. C.; Brown, M. R., Jr. In Cellulose and Wood-Chemistry and Technology; Conrad Schuerch, Ed.; Wiley Interscience: New York, 1988; pp 473-492.

15. Mayer, R.; Ross, P.; Weinhouse, H.; Amikam, D.; Volman, G.; Ohana, P. Calhoon, R. D.; Wong, H. C.; Emerick, A. W.; Benziman, M. Proc. Natl. Acad. Sci. U.S.A. 1991, 88, 5472-5476.
16. Newstead, S.; Ferrandon, S.; Iwata, S. Protein Sci. 2008, 17, 466-472.

17. Lin, F. C.; Brown, M. R., Jr.; Cooper, J. B.; Delmer, D. P. Science 1985, 230, 822 825.

18. Peng, L.; Kawagoe, Y.; Hogan, P.; Delmer, D. Science 2002, 295, 147-150.

19. Lai-Kee-Him, J.; Chanzy, H.; Müller, M.; Putaux, J.-P.; Imai, T.; Bulone, V. J. Biol. Chem. 2002, 277, 36931-36939.

20. Ohlsson, A. B.; Djerbi, S. Winzell, A.; Bessueille, L : Ståldal, V ; Li, X. Blomqvist, K.; Bulone, V.; Teeri, T. T.; Berglund, T. Protoplasma 2006, 228, 221-229.

21. Bessueille, L.; Sindt, N.; Guichardant, M.; Djerbis, S.; Teeris, T. T.; Bulone, V. Biochem. J. 2009, 420, 93-103.

22. Fujii, S.; Hayashi, T.; Mizuno, K. Plant Cell Physiol. 2010, 51, 294-301.

23. Langan, P.; Nishiyama, Y.; Chanzy, H. Biomacromolecules 2001, 2, 410-416.

24. Bureau, T. E.; Brown, M. R., Jr. Proc. Natl. Acad. Sci. U.S.A. 1987, 84, 6985-6989.

25. Kobayashi, S.; Hobson, L. J.; Sakamoto, J.; Kimura, S.; Sugiyama, J.; Imai, T.; Itoh, T. Biomacromolecules 2000, 1, 168-173.

26. Fort, S.; Boyer, V.; Greffe, L.; Davies, G. J.; Moroz, O.; Christiansen, L.; Schúlein, M.; Cottaz, S.; Driguez, H. J. Am. Chem. Soc. 2000, 122, 5429-5437.

27. Hiraishi, M.; Igarashi, K.; Kimura, S.; Wada, M.; Kitaoka, M.; Samejima, M. Carbohydr. Res. 2009, 344, 2468-2473.

28. Enebro, J.; Karlsson, S. Rapid Commun. Mass Spectrom. 2006, 20, 3693-3698.

29. Chan, T.-W. D.; Chan, P. K.; Tang, K. Y. Anal. Chim. Acta 2006, 556, 226-236.

30. Doelker, E.; Gurny, R.; Schurz, J.; Jánosi, A.; Matin, N. Powder Technol. 1987, 52, 207-213.

31. Kobayashi, S.; Kashiwa, K.; Kawasaki, T.; Shoda, S. J. Am. Chem. Soc. 1991, 113 3079-3084.

32. Samain, E.; Lancelon-Pin, C.; Ferigo, F.; Moreau, V.; Chanzy, H.; Heyraud, A. Driguez, H. Carbohydr. Res. 1995, 271, 217-226.

33. Marx-Figini, M. In Cellulose and Other Natural Polymer Systems; Brown, M. R., Jr., Ed.; Plenum Press: New York, 1982; pp 243-271.

34. Kimura, S.; Chen, H. P.; Saxena, I. M.; Brown, R. M. J.; Itoh, T. J. Bacteriol. 2001 $183,5668-5674$

35. Sarko, A. TAPPI 1978, 61, 59-61.

36. Hu, S.-Q.; Gao, Y.-G.; Tajima, K.; Sunagawa, N.; Zhou, Y.; Kawano, S.; Fujiwara, T.; Yoda, T.; Shimura, D.; Satoh, Y.; Munekata, M.; Tanaka, I.; Yao, M. Proc. Natl. Acad. Sci. U.S.A. 2010, 107, 17957-17961.

37. Wada, M.; Chanzy, H.; Nishiyama, Y.; Langan, P. Macromolecules 2004, 37, 8548-8555.

38. Saxena, I. M.; Brown, M. R., Jr.; Fevre, M.; Geremia, R. A.; Henrissat, B. J. Bacteriol. 1995, 177, 1419-1424.

39. Saxena, I. M.; Brown, M. R., Jr. Cellulose 1997, 4, 33-49.

40. Kudlicka, K.; Brown, M. R., Jr. Plant Physiol. 1997, 115, 643-656.

41. Knight, D. P.; Vollrath, F. Proc. R. Soc. London, Ser. B 1999, 266, 519-523.

42. Schramm, M.; Hestrin, S. J. Gen. Microbiol. 1954, 11, 123-129.

43. Ross, P.; Aloni, Y.; Weinhouse, H.; Michaeli, D.; Weinberger-Ohana, P.; Mayer R.; Benziman, M. Carbohydr. Res. 1986, 149, 101-117.

44. Ross, P.; Weinhouse, H.; Aloni, Y. Nature 1987, 325, 279-281.

45. Tamayo, R.; Tischler, A. D.; Camilli, A. J. Biol. Chem. 2005, 280, 33324-33330. 